\title{
Ruptura prematura de membranas de pretérmino en una población de bajo nivel socioeconómico. Resultados de un manejo con esteroides y betamiméticos
}

\author{
Rodrigo Cifuentes*; Hoover Canaval**; Jorge Londoño***
}

\begin{abstract}
RESUMEN: Se presentan resultados de 68 embarazadas con Ruptura Prematura de Membranas de Pretérmino (R.P.M.P.) sin infección ni trabajo de parto. La mitad $(n=34)$ evolucionó espontáneamente (grupo control). La otra mitad (grupo experimental), recibió a su ingreso esteroides (betametasona) más betamiméticos (terbutalina y fenoterol).
\end{abstract}

La edad gestacional, paridad, peso neonatal, puntaje de Apgar, infección puerperal y neonatal, mortalidad neonatal fue similar en ambos grupos. La incidencia de Síndrome de Dificultad Respiratoria (S.D.R.) en el grupo control fue de 70.5\%. En el grupo experimental de 32.3\% (chi cuadrado $=10.43 ; \mathrm{p}<0.01)$.

Los datos anteriores sugieren que en un manejo con esteroides y betamiméticos puede ser empleado selectivamente en R.P.M.P. en mujeres de bajo nivel socio-económico.

PALABRAS CLAVES: Ruptura Prematura de Membranas Pretérmino (R.P.M.P.), glucocorticoides, betamiméticos, Síndrome de Dificultad Respiratoria (S.D.R.).

SUMMARY: Preterm premature rupture of fetal membranes in a low socioeconomic population. Results of a management with corticosteroid and betamimetics.

We reporte the results of conservative management of 68 pregnant women with preterm premature rupture of the membranes (R.P.R.O.M.), without infection or labor. Thirty four (control group) were admitted and observed without medication. The other half (experimental group) received steroids (betamethasone) and betamimetics (terbutaline and fenoterol). The groups were evaluated for gestacional age at delivery, parity, mean birth weight, A pgar score, maternal or neonatel infection, neonatal mortality and respiratory distress syndrome (R.D.S.). Of these parameters, only the incidence of R.D.S. were statistically different in both groups: 70.5 percent in the control group and 32.3 percent in the experimental group $(\mathrm{X} 2=10.43 ; \mathrm{p}<0.01)$.

With these results, we suggest that conservative management with corticosteroids and betamimetics can be selectively employed in PPROM ocurring in women of low socioeconomic status.

KEY WORDS: Preterm premature rupture of the fetel membranes (PPROM); corticosteroids, betamimetics, respiratory distress syndrome (R.D.S.).

\section{Introducción}

El manejo perinatal de la embarazada con ruptura prematura de membranas de pretérmino (R.P.M.P.) permanece aún bastante controvertido. (1-3). Aunque la etiología de esta frecuente complicación del embarazo se desconoce, hay factores predisponentes que actúan modificando la elasticidad de las membranas ovulares facilitando su ruptura. Entre éstas podríamos citar infección local o intraamniótica, dilatación cervical, traumatismos, embarazo múltiple, polihidramnios (4-6).

La R.P.M.P. es una patología prevalente en todas las clases sociales. Sin embargo hay publicaciones que mencionan como es mayor mientras el nivel socio-económico

\footnotetext{
* Profesor Titular. Jefe Medicina Perinatal. Dpto. de Ginecoobstetricia. Facultad de Salud. Universidad del Valle, Cali.

** Residente de 3er año. Dpto. de Ginecoobstetricia. Universidad del Valle.

*** Residente de 3er. año. Dpto. de Ginecoobstetricia. Universidad Javeriana. Rotante por Medicina Perinatal en la Universidad del Valle.
}

sea más bajo (7). En nuestro medio, en el Hospital Universitario del Valle (H.U.V.) en Cali, la R.P.M. se encuentra asociada al $38.8 \%$ de todos los nacimientos de bajo peso (8).

El manejo conservador actualmente se acepta en muchos centros como método de elección en la R.P.M.P., lográndose disminuir la morbimortalidad perinatal en poblaciones de países desarrollados $(1,9)$. El propósito del presente trabajo fue evaluar resultados en el manejo conservador de la R.P.M.P. en una población de bajo nivel socioeconómico asistente al servicio de obstetricia del H.U.V.

\section{Pacientes y métodos}

Se presentan todas las pacientes que ingresaron con diagnóstico de ruptura prematura de las membranas ovulares entre 26 y 34 semanas de gestación desde el 1o. de noviembre de 1990 al 31 de octubre de 1991. El diagnóstico de R.P.M.P. se hizo usualmente por la presencia de líquido amniótico en la vagina o por una prueba positiva en papel de nitrazina (cambio de color de amarillo a azul).

Para ingresar al estudio además del diagnóstico de R.P.M.P. debía existir feto único, vivo, sin sospecha de 
infección, y sin actividad uterina de trabajo de parto al momento de la consulta.

Las embarazadas que cumplieran los requisitos para el estudio se asignaban alternativamente al grupo control, el cual evolucionó espontáneamente o al grupo experimental, el cual se manejó conservadoramente con dos fármacos, glucocorticoides y betamiméticos. El glucocorticoide utilizado fue betametasona en dosis de $12 \mathrm{mg}$. vía intramuscular que se repetían a las 24 horas. El betamimético utilizado fue terbutalina en infusión I.V. continua ( $5 \mathrm{mcg}$. minuto) por 24 horas, más fenoterol oral $(2.5 \mathrm{mg}$ cada 6 horas) durante otras 48 horas. Posteriormente, se suspendía la medicación y se dejaba evolucionar espontáneamente

Todas las pacientes tanto del grupo control como del grupo experimental, una vez fueron admitidas en el servicio de obstetricia, se les manejaba de acuerdo con los protocolos establecidos. En efecto, además del reposo en cama se les practicaba leucograma, estudio bacteriológico en vagina, monitoría fetal de no stress, y ultrasonido fetal, ya que una prueba de no stress no reactiva y/o la ausencia de movimientos respiratorios al estudio ultrasónico son altamente predictivos de infección fetal. Estas pruebas se repetían cada 48 horas. En caso de inicio espontáneo del trabajo de parto o sospecha de infección feto-materna, se interrumpía el embarazo por la vía obstétrica más indicada. Después del parto, los recién nacidos eran examinados por el servicio de neonatología.

Los parámetros neonatales evaluados fueron: Puntaje de Apgar al primero y quinto minuto de vida, hemograma, hemocultivo y presencia, o no de signos de Síndrome de Dificultad Respiratoria (S.D.R.).

Para análisis estadísticos el grupo control se comparó con el grupo experimental. Los grupos fueron evaluados básicamente para: edad gestacional al parto, presencia o no de S.D.R., puntaje de Apgar, mortalidad neonatal, infección puerperal neonatal así como horas transcurridas desde el ingreso hasta el parto. Se hicieron comparaciones utilizando pruebas de student o de chi cuadrado según los parámetros a comparar.

\section{Resultados}

Durante el período de estudio, 68 embarazadas con R.P.M.P., fueron aleatorizadas. La mitad $(n=34)$ evolucionó espontáneamente (grupo control). La otra mitad $(n=34)$ constituyó el grupo experimental. La edad gestacional al ingreso, paridad promedio, el peso de los recién nacidos en gramos, el Apgar al primero y quinto minuto se muestran en la Tabla 1. Como puede observarse, no hubo diferencias estadísticamente significativas entre los dos grupos estudiados.

Tabla 1

\section{EDAD GESTACIONAL, PARIDAD, PESO NEONATAL, PUNTAJE APGAR EN LOS GRUPOS ESTUDIADOS} MANEJO

\begin{tabular}{|lcc|}
\hline & $\begin{array}{c}\text { Expectante } \\
\text { (Control) }\end{array}$ & $\begin{array}{c}\text { Experimental } \\
\text { (esteroides y } \\
\text { betamiméticos) }\end{array}$ \\
\hline Edad gestacional (sem.) & 29.5 & 30.8 \\
Paridad (promedio) & 1.5 & 1.0 \\
Peso neonatal (en gs.) & 1.423 & 1.405 \\
Apgar al ler minuto < de 6 & 17 & 12 \\
Apgar al 5o. minuto < de 6 & 6 & 6 \\
\hline
\end{tabular}

No hubo diferencia estadística significativa entre los 2 grupos estudiados.
La incidencia de S.D.R., infección materna y neonatal así como la mortalidad neonatal se muestran en las tablas 2 y 3 . En ambas tablas puede observarse la incidencia de

Tabla 2

R.P.M. DE PRETERMINO. MORBI-MORTALIDAD MATERNA Y NEONATAL EN EL GRUPO CONTROL (MANEJO EXPECTANTE) Y EN EL GRUPO EXPERIMENTAL (ESTEROIDES MAS BETAMIMETICOS)

GRUPO

\begin{tabular}{|c|c|c|c|c|c|}
\hline \multirow[t]{2}{*}{$\begin{array}{l}\text { Control } \\
(n=34)\end{array}$} & & & \multicolumn{3}{|c|}{$\begin{array}{l}\text { Experimental } \\
(n=34)\end{array}$} \\
\hline & $\mathbf{n}$ & $\%$ & n & $\%$ & $\begin{array}{r}\text { p (chi - } \\
\text { cuadrado) }\end{array}$ \\
\hline S.D.R. & 24 & 70.5 & 11 & 32.3 & 0.01 \\
\hline Infección materna & 1 & 2.9 & 1 & 2.9 & N.S. \\
\hline Infección neonatal & 8 & 23.5 & 9 & 26.4 & N.S. \\
\hline Mortalidad neonatal & 4 & 11.7 & 3 & 8.8 & N.S. \\
\hline
\end{tabular}

Síndrome de Dificuitad Respiratoria en los dos grupos. De 34 pacientes en el grupo control, 24 recién nacidos (70.5\%) hicieron S.D.R. De 34 embarazadas en el grupo experimental, 11 recién nacidos (el $32.3 \%$ ) hicieron S.D.R. Una prueba de chi cuadrado (X2) nos permite demostrar que hay una relación de dependencia estadística entre el uso de esteroides y betamiméticos (grupo experimental) y la menor incidencia de S.D.R. $(X 2=10.43 ; p<0.01$, tabla 3$)$.

Tabla 3

R.P.M. DE PRETERMINO. MANEJO EXPERIMENTAL CON ESTEROIDES Y BETAMIMETICOS

\begin{tabular}{|l|l|lll|l|}
\hline \multicolumn{9}{|c|}{ S.D.R. } \\
\hline M & & SI & NO \\
A & EXPECTANTE & 24 & 10 & 34 & \\
N & & & & & X2 $=10.43$ \\
E & & & & & \\
J & EXPERIMENTAL & 11 & 23 & 34 & P 0.01 \\
O & & 35 & 33 & 68 & \\
& & 35 & & & \\
\hline
\end{tabular}

Obsérvese como hay menor incidencia de S.D.R. cuando se utiliza glucocorticoides y betamiméticos antenatalmente.

En relación con infección materna se presentó 1 caso en cada grupo de estudio. En ambos casos, se diagnosticó endometritis post parto y evolucionaron favorablemente con antibioticoterapia.

La infección neonatal tuvo una incidencia de 23.5 por ciento ( 8 casos) en el grupo control y de 26.4 por cinto ( 9 casos) en el grupo experimental. La mortalidad neonatal fue de 11.7 y 8.8 por ciento respectivamente para los dos grupos. Pruebas de chi cuadrado no mostraron diferencias estadísticamente significativas en la incidencia de infección puerperal o neonatal o en la mortalidad neonatal.

No hubo mortalidad materna en ninguno de los 2 grupos estudiados.

El tiempo transcurrido entre el inicio del tratamiento y el parto se muestra en la Tabla 4, en la cual puede observarse como en el grupo control el $56 \%$ de los nacimientos se produjo antes de cumplir 48 horas del ingreso. El $12 \%$ entre 
49 y 72 horas, y el $32 \%$ demoró más de 72 horas. En el grupo experimental los porcentajes fueron de 32,38 y $30 \%$ respectivamente.

\section{Tabla 4}

R.P.M. DE PRETERMINO. TIEMPO TRANSCURRIDO ENTRE EL INGRESO Y EL PARTO

\begin{tabular}{|lc|ccc|}
\hline & \multicolumn{2}{c|}{ Expectante } & \multicolumn{2}{c|}{ Experimental } \\
\hline Horas al parto & $\mathrm{n}$ & $\%$ & $\mathrm{n}$ & $\%$ \\
< de 48 & 19 & 56 & 11 & 32 \\
49 a 72 & 4 & 12 & 13 & 38 \\
+ de 72 & 11 & 32 & 10 & 30 \\
\hline
\end{tabular}

Es decir, cuando se utiliza esteroides y betamiméticos el tiempo transcurrido al parto es significativamente mayor particularmente por encima de las 48 horas.

\section{Discusión}

El anterior estudio nos sugiere que el manejo con glucocorticoides y betamiméticos, llamado por algunos "conservador", puede emplearse en la R.P.M.P., entre 26 y 34 semanas de gestación con el objetivo de disminuir el S.D.R., principal patología neonatal en la R.P.M.P., sin que por ello se incremente significativamente la morbilidad infecciosa materna o perinatal. El uso de los glucocorticoides en la R.P.M.P., creemos está ampliamente justificado ya que además de acelerar la maduración de los pulmones fetales a través de un incremento en la producción de fosfatióilcolina, se ha com- probado que tiene otros beneficios neonatales como son disminuir la incidencia de hemorragias periventriculares y enterocolitis necrotizante en el recién nacido prematuro. Sin embargo, para que los glucocorticoides produzcan su efecto inductor de la maduración sobre los alveolos pulmonares fetales debe transcurrir al menos 48 horas entre la administración de la segunda dosis y el nacimiento. Es por ello que además del glucocorticoide, debe utilizarse también un betamimético con el objetivo de retardar el inicio del parto por lo menos 48 a 72 horas después de la terapia esteroidea.

El riesgo teórico del manejo conservador con glucocorticoides es la infección materna y/o neonatal. En esta serie se presentó un caso de infección puerperal (2.9\%) en cada uno de los grupos estudiados. La infección neonatal tuvo una incidencia de $23.5 \%$ ( 8 casos) en el grupo control y $26.4 \%$ ( 9 casos) en el grupo experimental. Estos resultados demuestran que no existe ninguna diferencia en la incidencia de infección puerperal y neonatal entre las pacientes que recibieron esteroides y las que no lo recibieron.

En conclusión, se presentan los resultados neonatales favorables de un manejo conservador (esteroides y betamiméticos) de la R.P.M.P. en mujeres de bajo nivel socio-económico.

Para destacar, que cuando se maneja la R.P.M.P. con los fármacos ya mencionados se disminuye significativamente la incidencia de S.D.R. neonatal sin que se incremente la morbilidad materna o perinatal.

Finalmente sugerimos que toda paciente con R.P.M.P. entre 26 y 34 semanas de gestación, se maneje con glucocorticoides y betamiméticos las primeras 72 horas y luego se deje evolucionar espontáneamente.

\section{BIBLIOGRAFIA}

1. Graham LR., Gilstrap LC., Hauth JC., Kodack-Garza S., Conaster DE. Conservative management of patients with premature rupture of fetal membranes. Obstet. Gynecol. 1982; 59: 607-610.

2. Garite TJ., Keegan KA., Freeman RK., Nageotte MO. A randomized trial of ritodrine tocolysis versus expectant management in patients with premature rupture of membranes at 25 to 30 weeks of gestation. Am. J. Obstet. Gynecol. 1987; 157: 388-393.

3. Capeless E., Mead PB. Management of preterm rupture of membranes: Lack of a national consensus. Am. J. Obstet. Gynecol. 1987; 157: 11-12.

4. Naeye RL., Peters EC. Causes and consequences of premature rupture of fetal membranes. LANCET 1980; 1: 192-194.

5. Romero R., Quintero R., Oyarzun E. et al. Intraamniotic infection and the onset of labor in preterm premature rupture of the membranes. Am. J. Obstet. Gynecol. 1988; 159: 661-667.
6. Lenihan JP. Relationship of the antepartum pelvic examination to premature rupture of membranes. Am. J. Obstet. Gynecol. 1984; 63: 33-38.

7. Schreiber J., Benedetti T. Conservative management of preterm rupture of the fetal membranes in a low socioeconomic population. Am. J. Obstet. Gynecol. 1980; 136: 92-98.

8. Cifuentes R. Aspectos perinatales del nacimiento de pretérmino. En: Obstetricia de Alto Riesgo 3a. Ed. 1990; 113-128.

9. Andreyko JL., Chen CP., Shennan AT., Milligan JE. Results of conservative management of premature rupture of the membranes. Am. J. Obstet. Gynecol. 1984; 148: 600-604. 